\title{
Location Based Performance of WiMAX Network for QoS with Optimal Base Stations (BS)
}

\author{
Rakesh Kumar Jha, Idris Z. Bholebawa, Upena D. Dalal \\ Electronics and Communication Department Sardar Vallabhbhai National Institute of Technology, Surat, India. \\ Email: jharakesh.45@gmail.com, idris_bholebawa@yahoo.co.in, upena_dalal@yahoo.com \\ Received February 2 $2^{\text {nd }}, 2011$; Revised March 20 ${ }^{\text {th }}, 2011$; Accepted April $8^{\text {th }}, 2011$.
}

\begin{abstract}
In this paper we are given concept of our WiMAX (Worldwide Interoperability for Microwave Access) network performance for QoS monitoring and optimization solution for BS (Base Station) with multimedia application. In the communication sector, the optimal objective is to equate quality and cost. Due to its large coverage area, low cost of deployment and high speed data rates. WiMAX is a promising technology for providing wireless last-mile connectivity. Physical and MAC layer of this technology refer to the IEEE 802.16e standard, which defines 5 different data delivery service classes that can be used in order to satisfy Quality of Service (QoS) requirements of different applications, such as VoIP, videoconference, FTP, Web, etc. In this paper we have made six scenarios. Here two types of MAC layer QoS are used and they are UGS and rtPS having application of Voice over IP (VoIP) and MPEG respectively. Also the traffic priority for UGS is high as compared to rtPS. In each scenario the number of fixed nodes (Fixed Subscriber Stations) and Mobile nodes (Mobile Subscriber Stations) are different. To cover more nodes or if nodes are outside the coverage area more than one BS are required. All the simulation results based on optimized networks and area we have considered between Bhusaval and Jalgam.
\end{abstract}

Keywords: WiMAX, Physical Layer, MAC Layer, OPNET Modeler, QoS, Optimal Area

\section{Introduction}

Worldwide Interoperability for Microwave Access (WiMAX) is one of the most important broadband wireless technologies and is anticipated to be a viable alternative to traditional wired broadband techniques due to its cost efficiency. Being an emerging technology, WiMAX supports multimedia applications such as voice over IP (VoIP), voice conference and online gaming. It is necessary to provide Quality of Service (QoS) guaranteed with different characteristics. Therefore, an effective scheduling is critical for the WiMAX system. Many traffic scheduling algorithms are available for wireless networks, e.g. Round Robin, Proportional Fairness (PF) scheme and Integrated Cross-layer scheme (ICL). Among these conventional schemes, some cannot differentiate services, while some can fulfill the service differentiation with high-complexity implementation.

The IEEE 802.16 technology (WiMAX) is a promising alternative to $3 \mathrm{G}$ or wireless LAN for providing last-mile connectivity by radio link due to its large coverage area, low cost of deployment and high speed data rates. The standard specifies the air-interface between a Subscriber
Station (SS) and a Base Station (BS). The IEEE 802.162004 standard [1,2], also known as 802.16d, was published in October, 2004. This was further developed into the mobile WiMAX standard referred to as IEEE 802.16e2005 or 802.16 e [3] to support mobile users. IEEE 802.16 can be used not only as xDSL replacement for small business customers but also as a mobile internet access technology. There have been few studies focusing on performance evaluation of IEEE 802.16 WiMAX Networks using OPNET. Ramachandran et al. [4] studied performance evaluation of IEEE 802.16 for Broadband Wireless Access. However they used OPNET's DOCSIS models to simulate the IEEE 802.16 MAC. Rangel et al. [5] studied performance analysis of QoS scheduling in Broadband IEEE 802.16 Based Networks. Although using OPNET WiMAX models, they focused mainly on implementing their own scheduling algorithms. However, in the IEEE 802.16 standard, the scheduler is left open for implementation, thus creating an avenue for a healthy competition amongst manufacturers. While the standard defines the required procedures and messages for schedulers, it does not offer encouraging means to provide 
performance, reliability, or Quality of Service (QoS). Dang et al. [6] studied performance of scheduling algorithms for WiMAX networks. Some of their work is quite related with our works. However they focused mainly on implementing some existing scheduling algorithms.

The purpose of this study was to examine a case of QoS deployment over a cellular WiMAX network and to examine the capability of a WiMAX network to deliver adequate QoS to voice and data applications. The methodologies taken include creating the WiMAX network, deploying the required applications, deploying QoS configurations within the WiMAX last-mile, adjusting the QoS configurations within the WiMAX cells to meet voice requirements, and further adjusting the QoS configurations to improve data application performance, without degrading the performance of voice [7]. This topic was identified as being importance to researcher and manufacturers in providing them the necessary background for their works.

This paper discusses about the performance of the MAC Layer by applying different QoS applications using OPNET Modeler Simulation Tool.

The paper is organized as follows. Section 2 explains the QoS in IEEE 802.16. Section 3 provides an overview of VoIP including R-Score and MOS. Section 4 provides the design of system model. The results are presented in Section 5. Finally, Section 6 concludes the paper.

\section{Quality of Service (QoS) in IEEE 802.16}

Originally, four different service types were supported in the 802.16 standard: UGS, rtPS, nrtPS and BE [1,8].

\subsection{The UGS (Unsolicited Grant Service)}

Is similar to the CBR (Constant Bit Rate) service in ATM, which generates a fixed size burst periodically. This service can be used to replace T1/E1 wired line or a constant rate service. It also can be used to support real time applications such as VoIP or streaming applications. Even though the UGS is simple, it may not be the best choice for the VoIP in that it can waste bandwidth during the off period of voice calls.

\subsection{The rtPS (Real-Time Polling Service)}

Is for a variable bit rate real-time service such as VoIP. Every polling interval, BS polls a mobile and the polled mobile transmits bw-request (bandwidth request) if it has data to transmit. The BS grants the data burst using UL-MAP-IE upon its reception.

\subsection{The nrtPS (Non-Real-Time Polling Service)}

Is very similar to the rtPS except that it allows contention based polling.

\subsection{The BE (Best Effort)}

Service can be used for applications such as e-mail or FTP, in which there is no strict latency requirement. The allocation mechanism is contention based using the ranging channel. Another service type called ertPS (Extended rtPS) [7] was introduced to support variable rate real-time services such as VoIP and video streaming. It has an advantage over UGS and rtPS for VoIP applications because it carries lower overhead than UGS and rtPS.

The details have given in Table 1.

\section{WiMAX Radio Planning for Simulation}

WiMAX deployment with optimization is not easy task so we have done survey between Bhusawal, Balwadi and Jalgam. In this particular area we also considered about number of users. Since we know that WiMAX coverage is $50 \mathrm{~km}$ in radius but when we have take practical consideration it's up to 25 to $30 \mathrm{~km}$. We have also observed that one directional antenna and one Omni-directional antenna has been required for last mile operation or for total coverage. The area with location and required range and BS is given in Figure 1.

\section{Model for Implementation}

In this experiment, we used OPNET Modeler version

Table 1. Service flows supported in WiMAX.

\begin{tabular}{|c|c|c|}
\hline Service-Flow Designation & Defining QoS Parameters & Application Examples \\
\hline Unsolicited-Grant Service (UGS) & $\begin{array}{l}\text { Maximum sustained rate, Maximum latency tolerance, } \\
\text { Jitter tolerance }\end{array}$ & $\begin{array}{l}\text { Voice over IP (VoIP) without silence } \\
\text { suppression }\end{array}$ \\
\hline Real-Time Polling Service (rtPS) & $\begin{array}{l}\text { Minimum reserved rate, Maximum sustained rate, } \\
\text { Maximum latency tolerance, Traffic priority }\end{array}$ & $\begin{array}{l}\text { Streaming audio and video, MPEG } \\
\text { (Motion Picture Experts Group) encoded }\end{array}$ \\
\hline Non-Real-Time Polling Service (nrtPS) & $\begin{array}{l}\text { Minimum reserved rate, Maximum sustained rate, } \\
\text { Traffic priority }\end{array}$ & File Transfer Protocol (FTP) \\
\hline Best-Effort Service (BE) & Maximum-sustained rate, Traffic priority & Web-browsing, Data transfer \\
\hline Extended Real-Time Polling Service (ErtPS) & $\begin{array}{l}\text { Minimum reserved rate, Maximum sustained rate, Maxi- } \\
\text { mum latency tolerance, Jitter tolerance, Traffic priority }\end{array}$ & VoIP with silence suppression \\
\hline
\end{tabular}




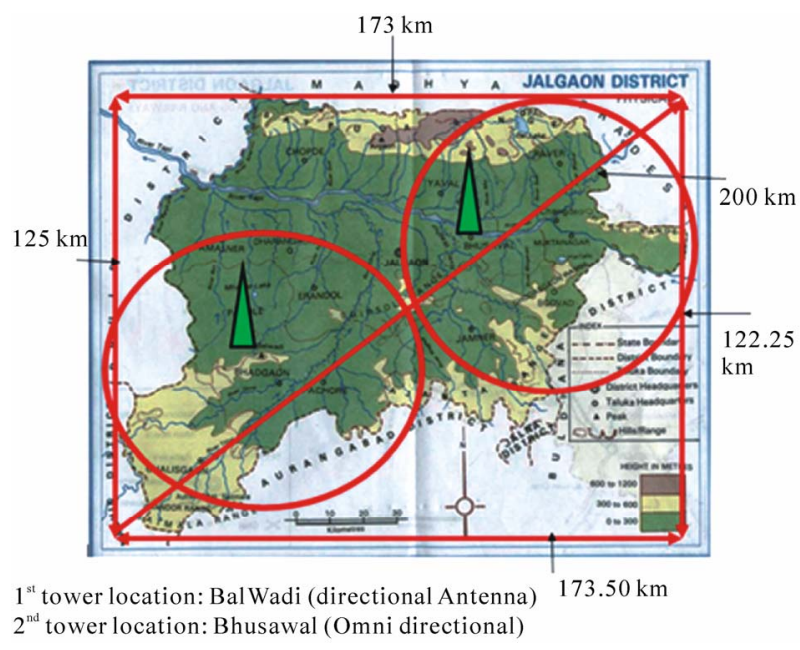

Figure 1. Theoretical overview of proposed Network with optimal BS.

14.5 with WiMAX Module capability [9]. We designed six scenarios including one scenario with one BS only and rest of five scenarios has two base stations between Jalgam and Bhusawal district. This model also provides the performance idea with fixed and mobile client stations.

\section{Network Model}

In our proposed location based networks have six different models. The clients is increased but whole area is constant i.e. area under supervision is Jalgam and Bhusawal. Following are the six different networks model those are created by us in OPNET MODELER 14.5.

Scenario 1 is shown in Figure 2 have placed one Base Station (BS) near Jalgaon (Maharashtra, India) which interfaces with four nodes includes 2 fixed nodes and 2 mobile nodes. In this scenario the WiMAX parameters which we have used are listed in Table 2.

Scenario 2 is shown in Figure 3, in this scenario four fixed nodes and four mobile nodes are present. Also to cover large distance we have simply increase the number of base station by two. The other base station in kept near Bhusaval (Maharashtra, India). The distance between Jalgaon and Bhusaval is near about $23 \mathrm{kms}$, since WiMAX base station practically covers up to $30 \mathrm{kms}$ (Practically).

Scenario 3 is shown in Figure 4, in this scenario six fixed nodes and six mobile nodes are used, which are covered the two BSs just like scenario 2. The WiMAX parameters are same.

Scenario 4 is shown in Figure 5, in this scenario 8 fixed nodes and 8 mobile nodes are used. Just like scenario 2 and scenario 3 the total 16 nodes are covered by the two BSs. Here we have placed all mobile nodes

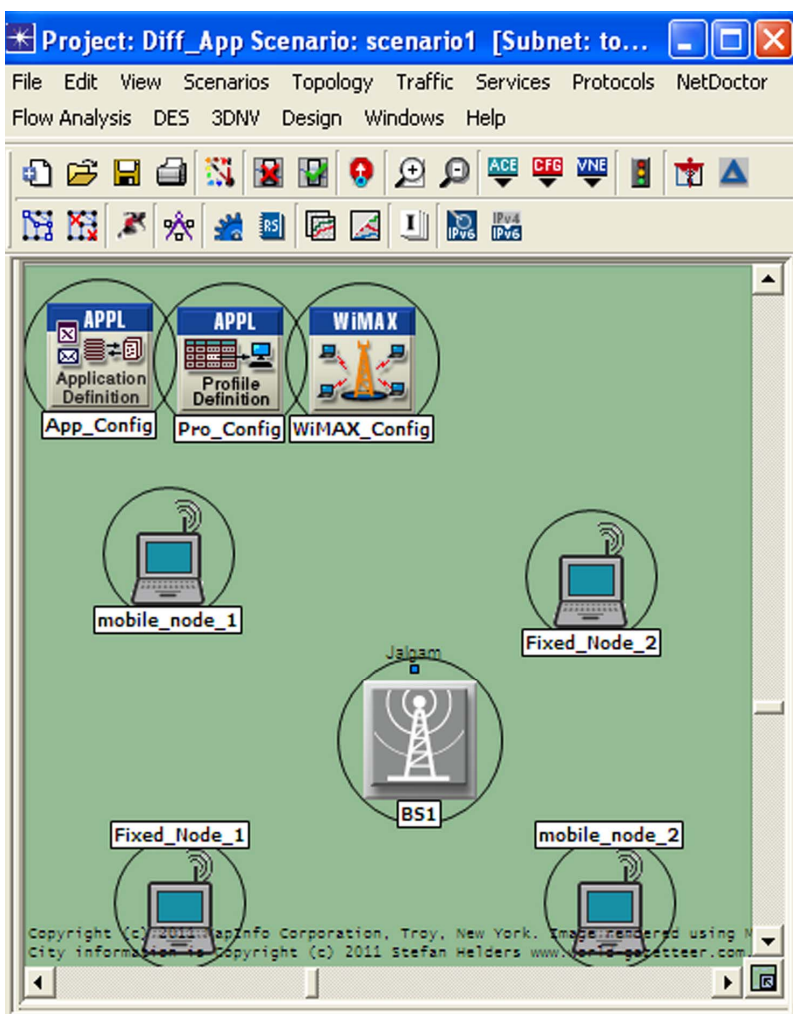

Figure 2. Network model first.

Table 2. Simulation parameters.

\begin{tabular}{lc}
\hline \multicolumn{1}{c}{ Efficiency Mode } & Mobility and Ranging Enabled \\
\hline $\begin{array}{l}\text { MAC Service Class } \\
\text { Definition (QoS) }\end{array}$ & 1) UGS \\
e.g. VoIP (IP Telephony) & 2) rtPS \\
Modulation Technique & e.g. MPEG (High Resolution Video) \\
Number of Subcarriers & Wireless OFDM \\
Bandwidth & 2048 \\
Duplexing Technique & $20 \mathrm{MHz}$ \\
Frame Duration & $\mathrm{TDD}$ \\
Symbol Duration & $1 \mathrm{~ms}$ \\
\hline
\end{tabular}

nearer to the Base Stations (BSs) and fixed nodes far to the BSs but in coverage area of BSs.

Scenario 5 is shown in Figure 6, in this scenario 9 fixed nodes and 9 mobile nodes are used that means total 18 nodes are used which are simply covered by 2 BSs, and the WiMAX parameters are again same.

Scenario 6 is shown in Figure 7, in this scenario we have simply increased nodes to 20 which include 10 fixed nodes and 10 mobile nodes, and again it is covered by the same 2 BSs with the same WiMAX parameters. 


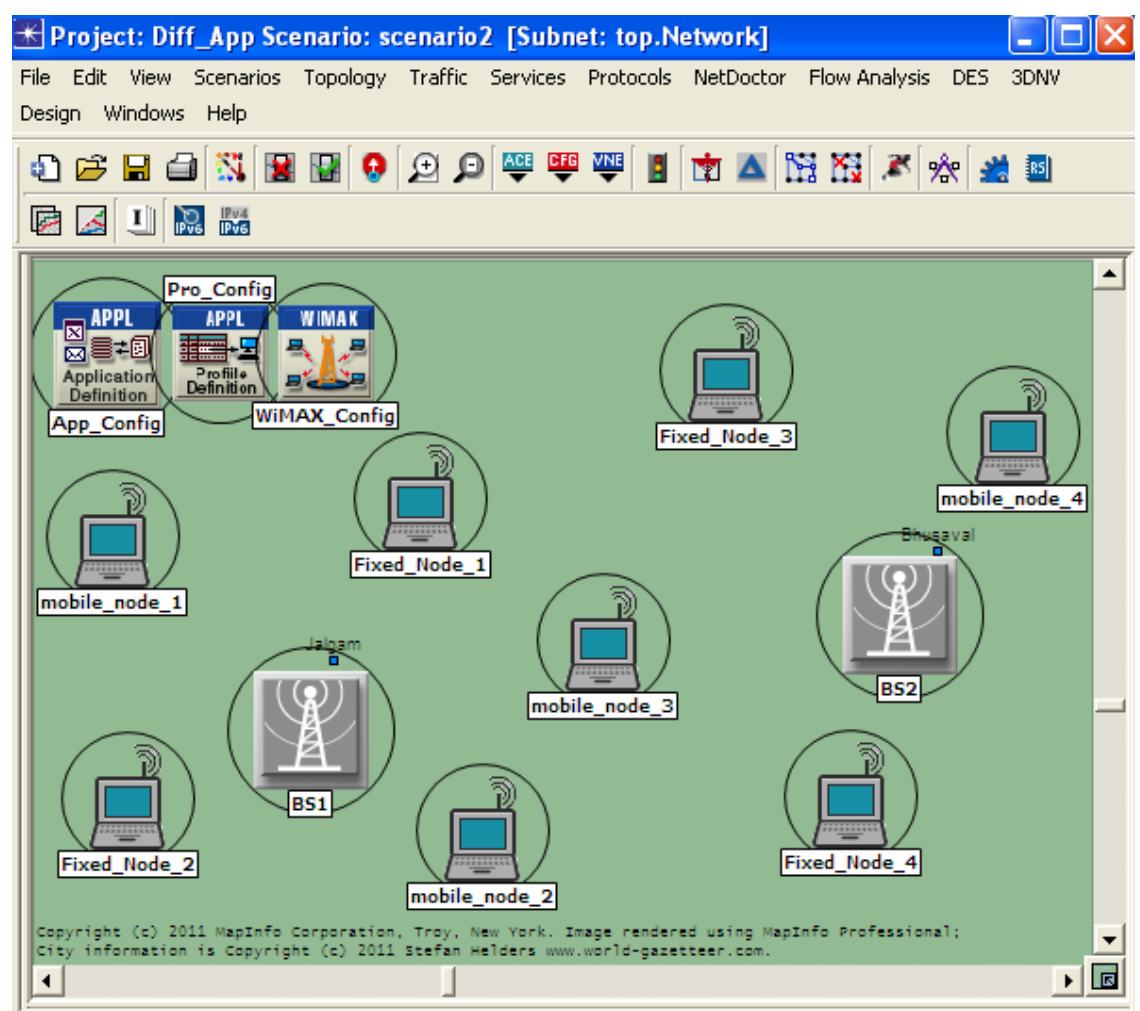

Figure 3. Network model second.

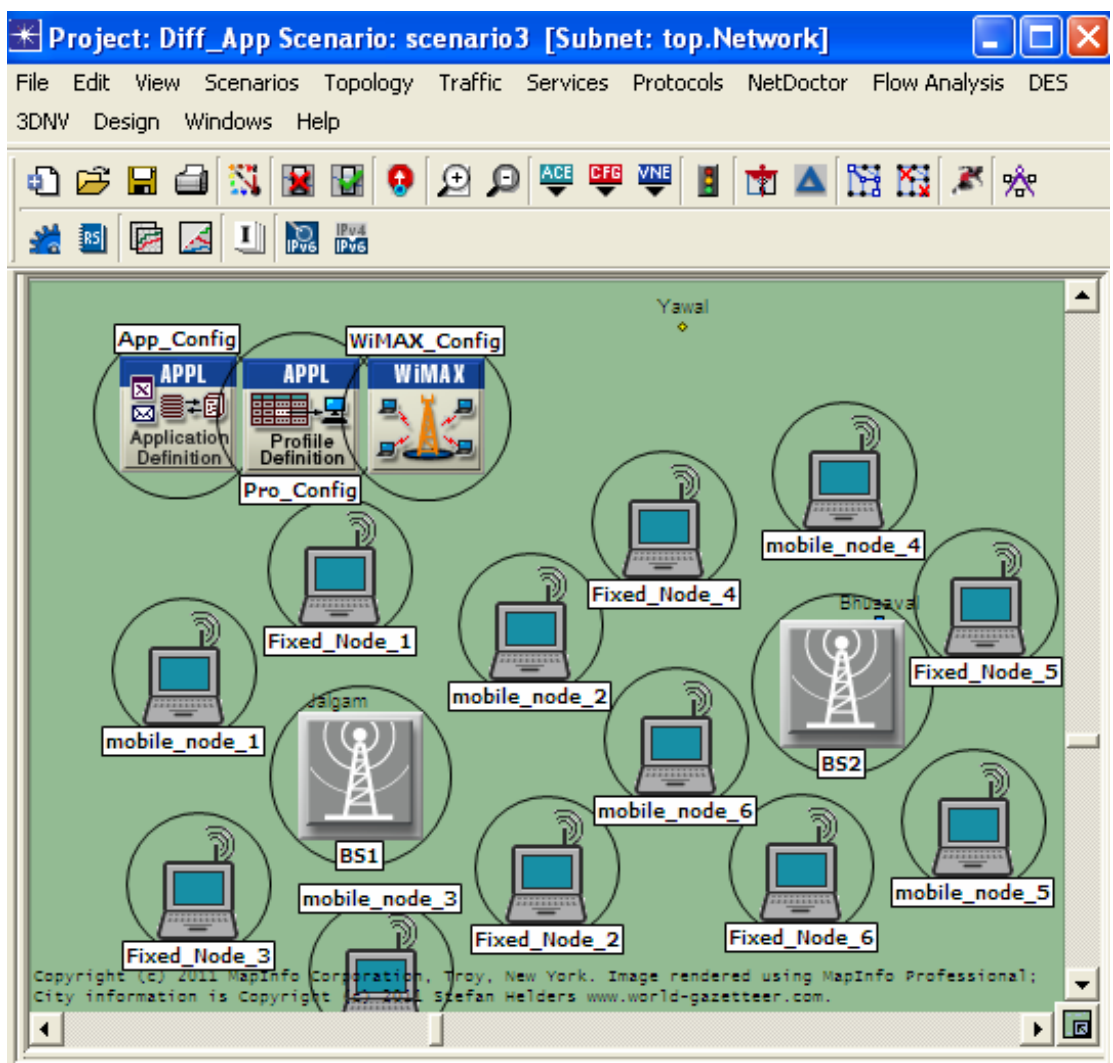

Figure 4. Network model third. 


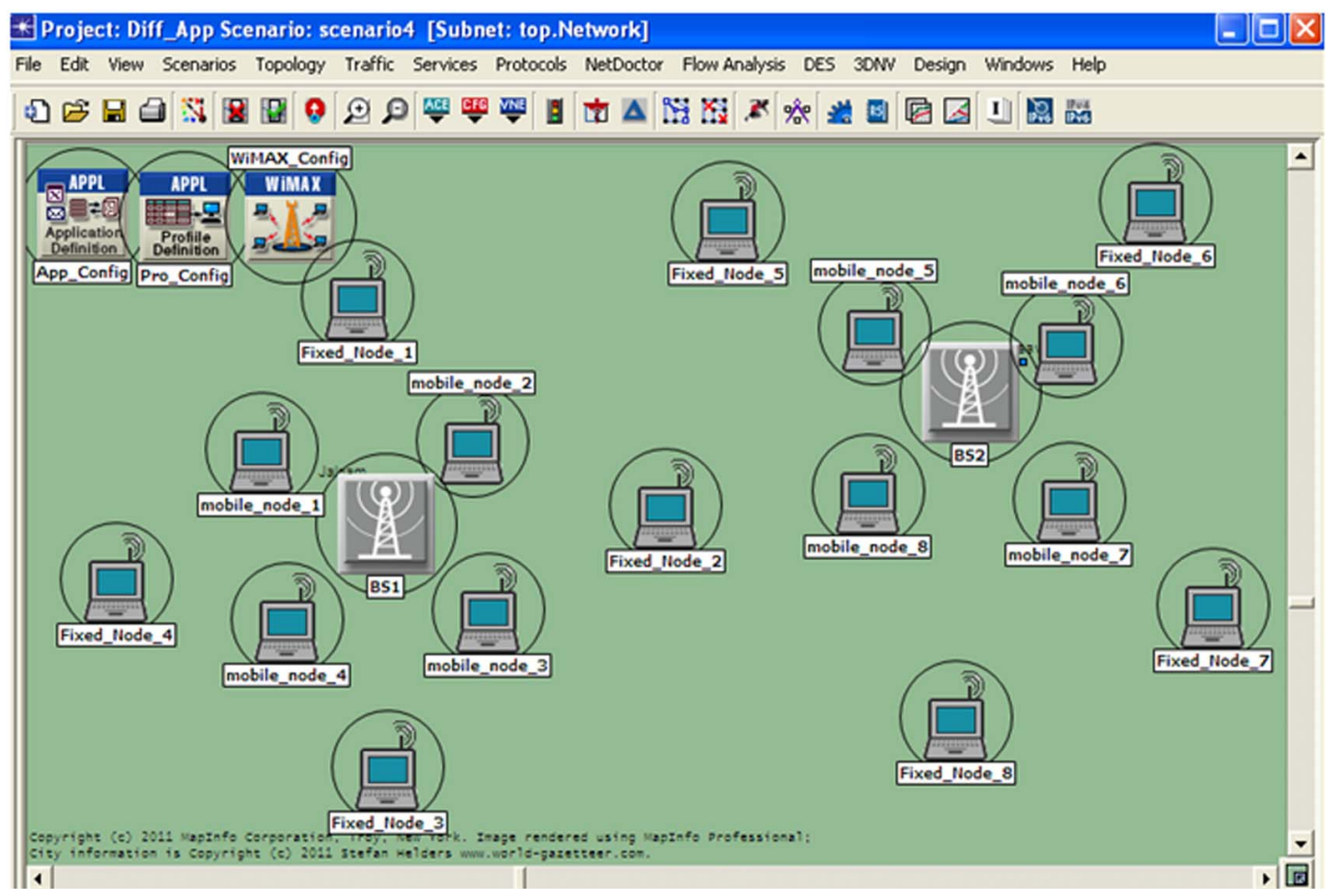

Figure 5. Network model fourth.

ॠ Project: Diff_App Scenario: scenario5 [Subnet: top.Network]

File Edit View Scenarios Topology Traffic Services Protocols NetDoctor Flow Analysis DES 3ONV Design Windows Help

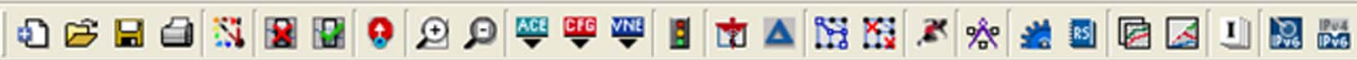

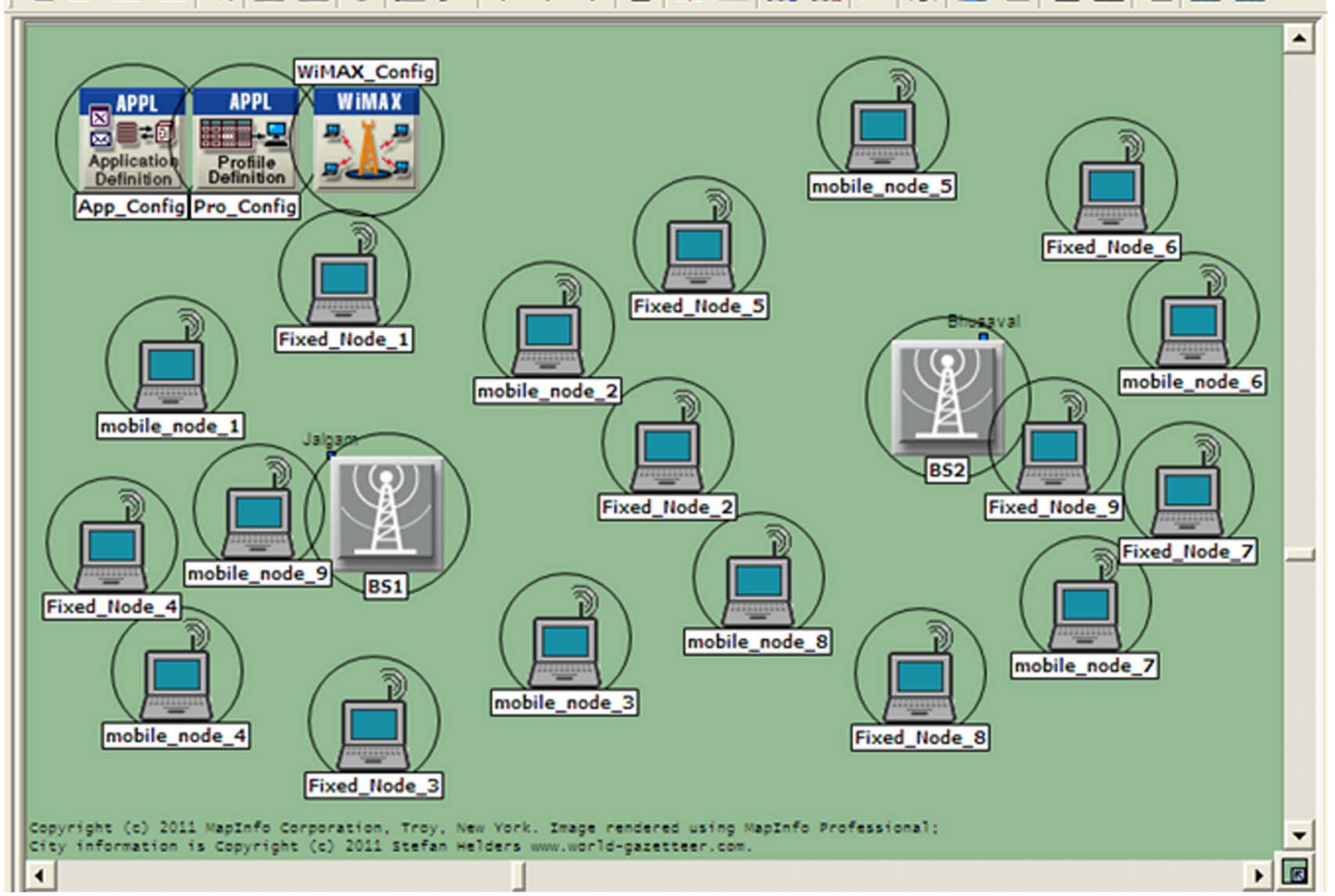

Figure 6. Network model five. 


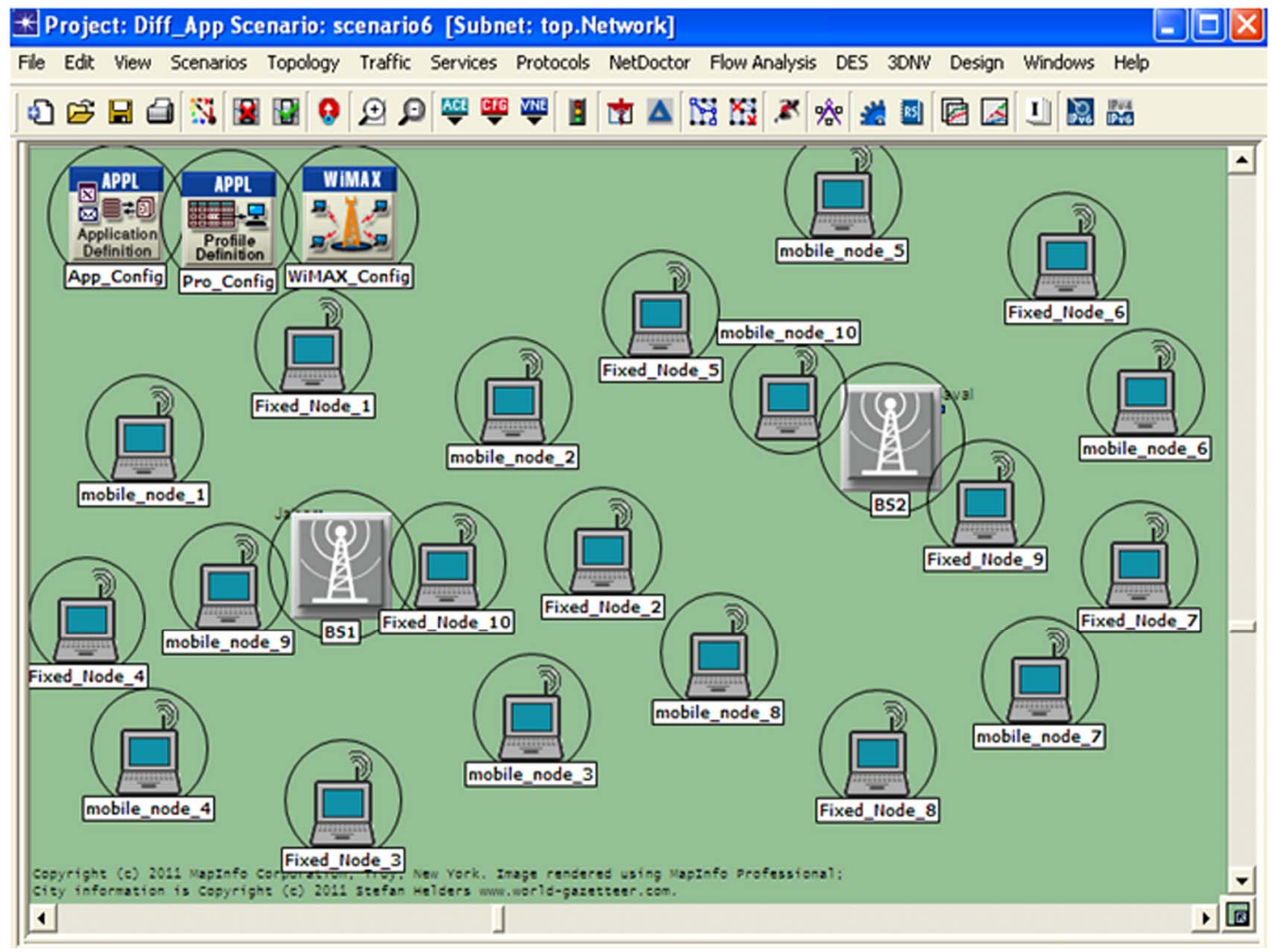

Figure 7. Network model six.

Parameter Involved in all scenarios are given below. Parameter associated with WiMAX configuration is given in Figure 8.

The WiMAX configuration object includes the following attributes.

- Contention Parameters

- Efficiency Mode

- MAC Service Class Definitions

- OFDM(A) PHY Profiles

- SC PHY Profiles

On base station node models and subscriber station node models, all of the parameters needed to specify WiMAX configuration are included in the WiMAX Parameters compound attribute. Figure 8 shows the WiMAX Parameter attributes.

Figure 9 and Figure 10 indicates the individual attributes for WiMAX Configuration.

As shown in Figure 9 the contention parameter is set in uniformity with minimum and maximum outcome of 1 and 10 respectively. This attribute characterizes the contention behavior of bandwidth requests. The second attribute is of Efficiency Mode. Here there are four types of Efficiency Modes as follows:

- Efficiency Enabled

- Framing Module Enabled
- Physical Layer Enabled

- Mobility and Ranging Enabled

Efficiency Mode attributes is set in Mobility and Ranging Enabled mode; in this mode all WiMAX model features are enabled. WiMAX MAC service class definition is described in Figure 10.

As described in figure two types of MAC Layer Quality of services (QoS) UGS and rtPS are used, with maximum sustained traffic rate of $5 \mathrm{Mbps}$ and minimum sustained traffic rate of 1 Mbps for UGS and with maximum sustained traffic rate of $1 \mathrm{Mbps}$ and minimum sustained traffic rate of $0.5 \mathrm{Mbps}$ for rtPS scheduling types.

Other WiMAX parameters of OFDM PHY Profiles and SC PHY Profiles are described in Table 2.

Parameter associated with WiMAX Subscriber Station (SS) is given in Figure 11.

Here for Fixed Node the Multipath Channel Model is kept as ITU Pedestrian A and Mobile Node is kept as ITU Vehicular A form. For both Fixed and Mobile node the Base Station is distance based, it means that the SS Node can communicate with a BS if the SS is in a range of that particular a BS. Downlink and Uplink Service Flows are kept according to IEEE 802.16e [3] standards.

The base station nodes have a similar set of WiMAX parameters that includes attributes for base station (instead 


\section{* (WimaX_Config) Attributes}

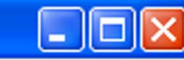

\begin{tabular}{|c|c|c|c|}
\hline \multicolumn{4}{|l|}{ Type: Utilities } \\
\hline Attribute & \multicolumn{3}{|l|}{ Value } \\
\hline (?) :- name & \multicolumn{3}{|c|}{ WiMAX_Confia } \\
\hline (?) $\Phi$ AMC Profile Sets Definitions & \multicolumn{3}{|l|}{ (...) } \\
\hline (?) $\mp$ Contention Parameters & \multicolumn{3}{|l|}{$(\ldots)$} \\
\hline (?) Efficiency Mode & \multicolumn{3}{|c|}{ Mobility and Rianging Enabled } \\
\hline (?) $\mp M A C$ Service Class Definitions & \multicolumn{3}{|l|}{$[\ldots]$} \\
\hline (?) \pm OFDM PHY Profiles & \multicolumn{3}{|l|}{$[\ldots]$} \\
\hline$\mp$ SC PHY Profiles & \multicolumn{3}{|l|}{$[\ldots]$} \\
\hline & & \multirow{2}{*}{\multicolumn{2}{|c|}{$\begin{array}{r}\Gamma \text { Adyanced } \\
\Gamma \text { Apply to selected objects }\end{array}$}} \\
\hline (?) & Filter & & \\
\hline \multicolumn{2}{|l|}{$Г$ Exact match } & $\underline{\underline{Q} K}$ & Cancel \\
\hline
\end{tabular}

Figure 8. WiMAX configurations.
(?) $\mathrm{C}$ Contention Parameters
(2) Number of Retries
(?) Efficiency Mode
(?) \pm MAC Service Class Definitions
(3) \pm OFDM PHY Profiles
(?) \pm SC PHY Profiles

(...) uniform_int $(1,10)$ Mobility and Rangina Enabled

Efficiency Enabled

Framing Module Enabled

Physical Layer Enabled Mobility and Ranaing Enabled

Figure 9. WiMAX configuration attributes.

\begin{tabular}{|c|c|c|}
\hline \multicolumn{2}{|c|}{ (?) $\oplus$ Contention Parameters } & \multirow{2}{*}{$\begin{array}{l}\text { (...) } \\
\text { Mobility and Ranging Enabled }\end{array}$} \\
\hline \multicolumn{2}{|c|}{ (?) Efficiency Mode } & \\
\hline \multicolumn{2}{|r|}{ (3) $-\mathrm{MAC}$ Service Class Definitions } & $(\ldots)$ \\
\hline (?) & Number of Rows & 2 \\
\hline \multicolumn{2}{|r|}{$\begin{array}{l}\text { Row } 0 \\
\square \text { Romer of }\end{array}$} & \\
\hline (?) & Service Class Name & Gold \\
\hline (?) & Scheduling Type & UGS \\
\hline (?) & Maximum Sustained Traffic Rate (b... & $5 \mathrm{Mbps}$ \\
\hline (?) & Minimum Reserved Traffic Rate (bps) & $1 \mathrm{Mbps}$ \\
\hline (?) & Maximum Latency (milliseconds) & 30.0 \\
\hline (?) & - Maximum Traffic Burst (bytes) & 0 \\
\hline (?) & . Traffic Priority & Not Used \\
\hline (?) & ... Unsolicited Poll Interval (milliseconds) & Auto Calculated \\
\hline \multicolumn{2}{|r|}{$\boxminus$ Row 1} & \\
\hline (?) & Service Class Name & Silver \\
\hline (?) & Scheduling Type & rtPS \\
\hline (3) & Maximum Sustained Traffic Rate (b... & $1 \mathrm{Mbps}$ \\
\hline (?) & Minimum Reserved Traffic Rate (bps) & $0.5 \mathrm{Mbps}$ \\
\hline (?) & Maximum Latency [milliseconds] & 30.0 \\
\hline (3) & Maximum Traffic Burst (bytes) & 0 \\
\hline (?) & Traffic Priority & Not Used \\
\hline (?) & Unsolicited Poll Interval (milliseconds) & Auto Calculated \\
\hline \multicolumn{2}{|r|}{ (?) $\oplus$ OFDM PHY Profiles } & $(\ldots)$ \\
\hline \multicolumn{2}{|c|}{ (?) \pm SC PHY Profiles } & {$[\ldots]$} \\
\hline
\end{tabular}

Figure 10. WiMAX configuration attributes for MAC service class definition. 


\begin{tabular}{|c|c|c|c|c|}
\hline *1 & ixed_Node_1) Attributes & & $\square \square$ & $x$ \\
\hline Type & workstation & & & \\
\hline & ttribute & Value & & $\Delta$ \\
\hline (3) & PHY Profile Type & OFDM & & \\
\hline (2) & - SS Parameters & $(\ldots)$ & & \\
\hline (2) & BS MAC Address & Distance Based & & \\
\hline (2) & $\oplus$ Downlink Service Flows & $(\ldots)$ & & \\
\hline (2) & Ð Uplink Service Flows & {$[\ldots]$} & & \\
\hline (3) & Multipath Channel Model & ITU Pedestrian A & & \\
\hline & \pm Pathloss Parameters & {$[\ldots]$} & & \\
\hline (2) & Ranging Power Step (mW) & 0.25 & & \\
\hline (2) & \pm Timers & Default & & \\
\hline (2) & - Contention Ranging Retries & 16 & & \\
\hline (2) & \pm Mobility Parameters & Default & & \\
\hline & Ð HARQ Parameters & $(\ldots)$ & & \\
\hline (2) & Piggyback BW Request & Enabled & & \\
\hline (3) & - CQICH Period & 3 & & \\
\hline (2) & - Contention-Based Reservation Tim... & 16 & & \\
\hline (2) & Request Retries & 16 & & \\
\hline & Applications & & & 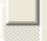 \\
\hline & $\mathrm{H} 323$ & & & \\
\hline & CPU & & & \\
\hline & Client Address & Auto Assigned & & \\
\hline & IP Multicasting & & & - \\
\hline
\end{tabular}

Figure 11. WiMAX subscriber station parameters.

of subscriber station) operation. The three-sector base station has three sets of attributes under WiMAX Parameters, one for each sector.

Parameter associated with WiMAX Application configurations is given in Figure 12.

A user profile is built using various application definitions. An application definition specifies an application with parameters. An application may have tasks and a task may have multiple phases. A phase can have many requests and responses.

Here as described earlier two types of services are used Audio and Video application services. Audio application includes IP Telephony and Video application includes High Resolution Video Conferencing.

Parameter associated with WiMAX profile configurations is given in Figure 13.

\begin{tabular}{|c|c|c|}
\hline \multicolumn{3}{|c|}{ Type: utility } \\
\hline & Attribute & Value \\
\hline (3) & ". name & ApD_Confia \\
\hline (?) & - Application Definitions & {$[\ldots]$} \\
\hline & Number of Rows & 2 \\
\hline & $\oplus$ audio_app & $\ldots$ \\
\hline & Ð video_app & $\ldots$ \\
\hline & $\mp \operatorname{MOS}$ & \\
\hline (?) & $\doteq$ Voice Encoder Schemes & All Schemes \\
\hline
\end{tabular}

Figure 12. WiMAX Application configuration.

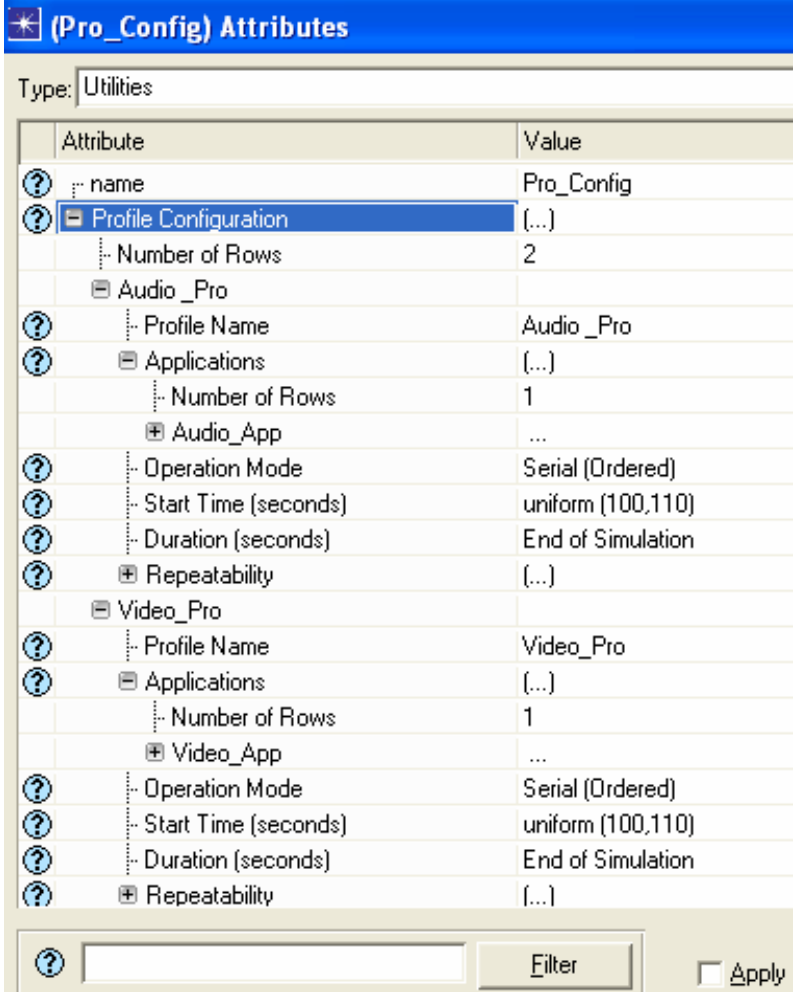

Figure 13. WiMAX profile configurations with Application.

A profile describes user activity over a period of time. A profile consists of many different applications. In order to configure a particular workstation to use a particular 
profile, we need to set this profile name on the "Application Supported Profiles" attribute on the workstation.

\section{Performance Analysis of Delay and Throughput}

In this research, we have divided our work into six different scenarios with the help of OPNET Modeler. Here two types of MAC layer QoS are used and they are UGS and rtPS having application of Voice over IP (VoIP) and MPEG respectively. Also the traffic priority for UGS is high as compared to rtPS. In each scenario the number of fixed nodes (Fixed Subscriber Stations) and Mobile nodes (Mobile Subscriber Stations) are different. To cover more nodes or if nodes are outside the coverage area more than one BS are required. Through different scenario we have compare the throughput and delay with respect to time. The simulation parameters used in this model are listed in Table 2.

\section{Results}

Here the global analyses of all the scenarios are done and the comparison of average throughput (packets/sec), average load (packets/sec) and average delay (sec) are given.

Figure 14 indicates the comparison between Throughputs (packets/sec) Vs Simulation Time of all six scenar- ios. In all scenarios every SS can communicate simultaneously with each other through base station. Simulation time is taken as 60 seconds, after simulation we have observed that throughput of scenario 1 (2 fixed and 2 mobile) is nearly about 0.5 packets/sec, scenario 2 (4 fixed and 4 mobile) have 0.9 packets/sec, scenario 3 (6 fixed and six mobile) have 1.1 packets/sec, scenario 4 (8 fixed and 8 mobile) have 1.4 packets/sec, scenario 5 (9 fixed and 9 mobile) have 1.9 packets/sec and scenario 6 (10 fixed and 10 mobile) have 2.4 packets/sec.

Figure 15 indicates the comparison between Loads (packets/sec) Vs Simulation Time in each scenario. Scenario 1 have 0.14 packets/sec, scenarios 2 have 0.22 packets/sec, scenario 3 have 0.21 packets/sec, scenario 4 have 0.17 packets /sec, scenario 5 have 0.20 packets/sec, and scenario 6 have the highest load of about 0.24 packets/sec.

Figure 16 indicates the result between Delay (sec) Vs. Simulation Time. We have seen that delay of scenario 1 has $0.0032 \mathrm{sec}$ throughout the simulation time (constant delay). Similarly scenario 2 have 0.0017 sec initially and remains constant at $0.0015 \mathrm{sec}$, scenario 3 delay is vary from 0.0048 to $0.0038 \mathrm{sec}$, scenario 4 varies from 0.0015 to $0.0034 \mathrm{sec}$, scenario 5 varies between 0.0058 to 0.0054 sec and scenario 6 varies from 0.0031 to 0.0017 sec.

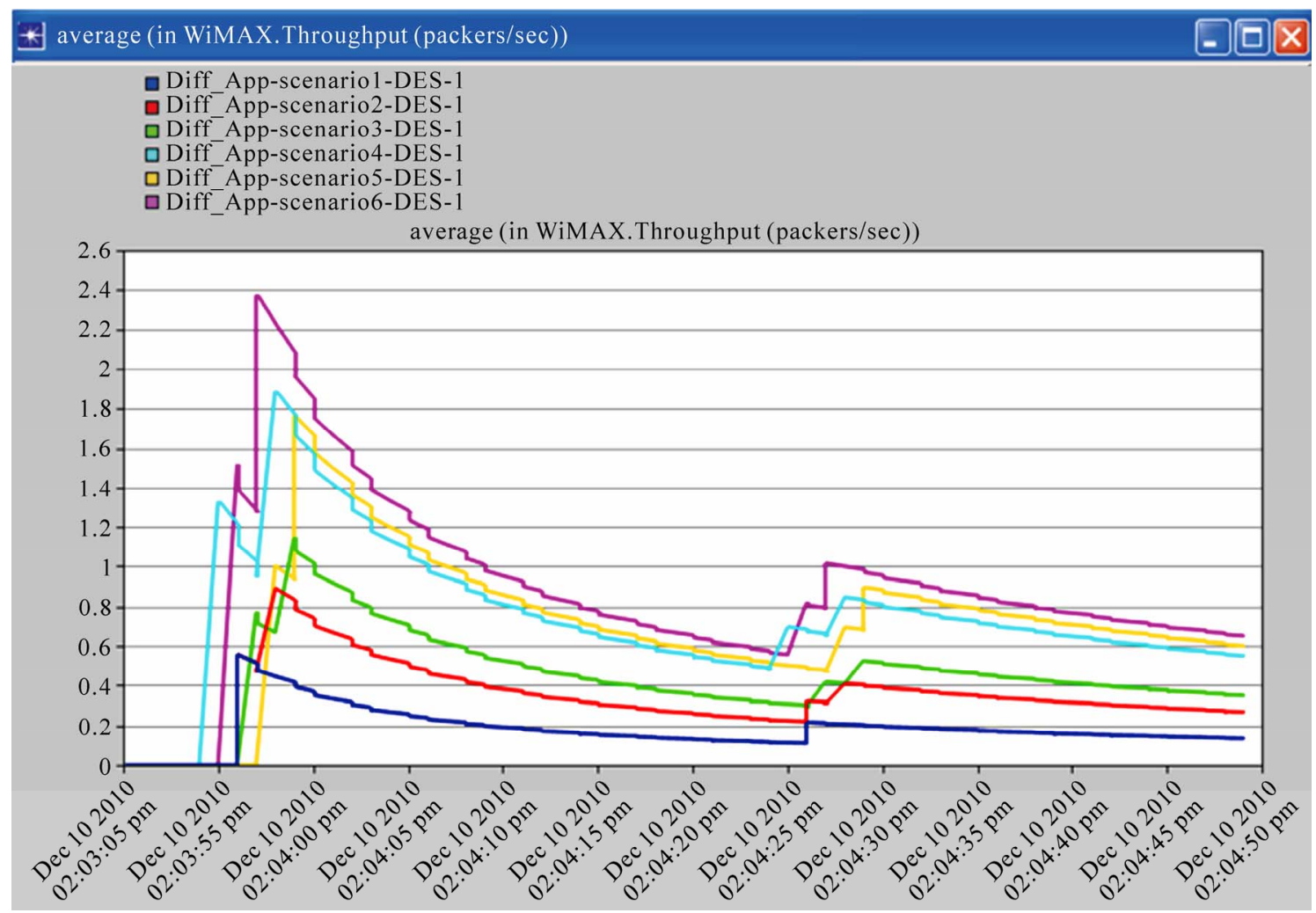

Figure 14. Throughput vs. simulation time. 


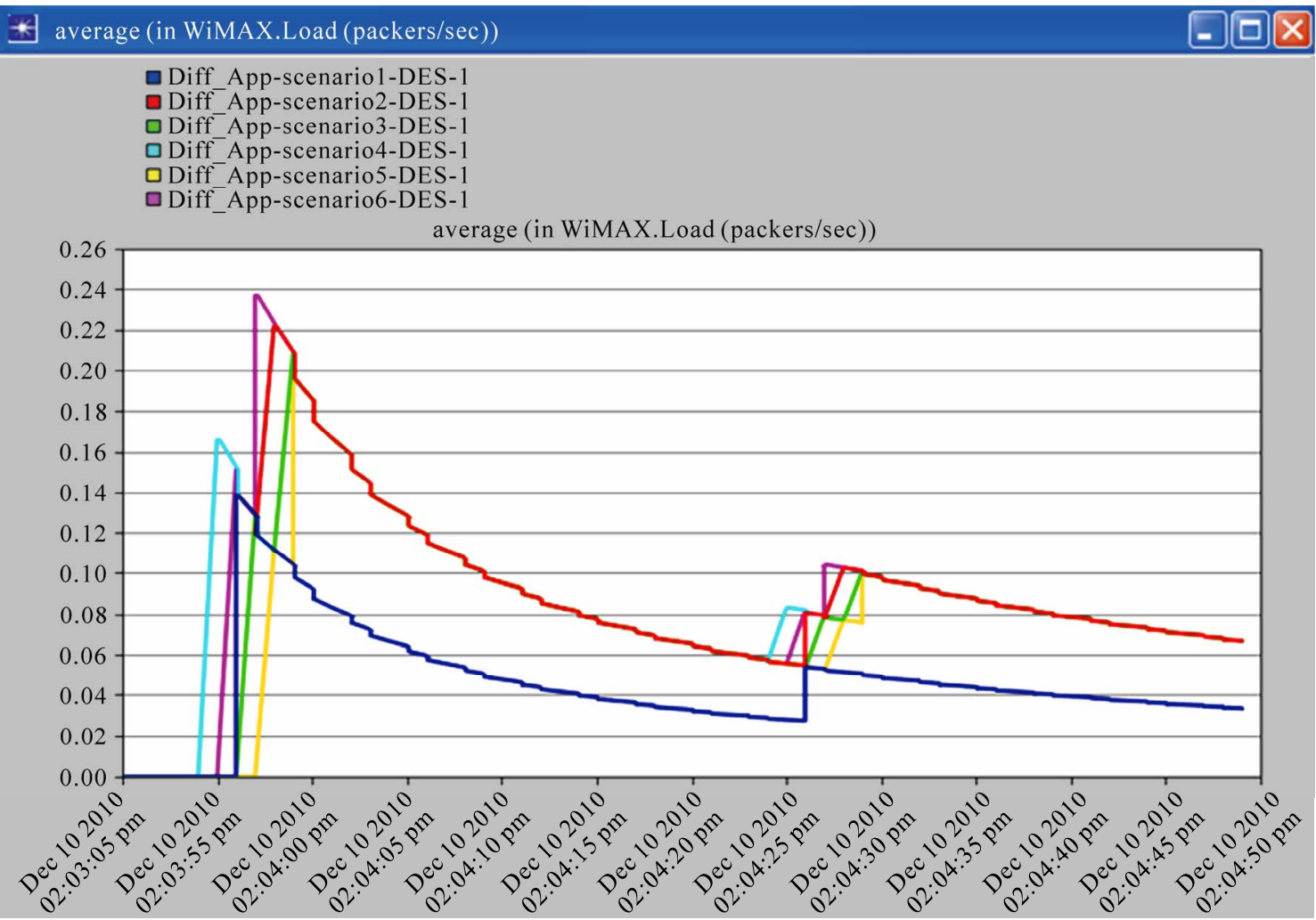

Figure 15. Load vs simulation time.

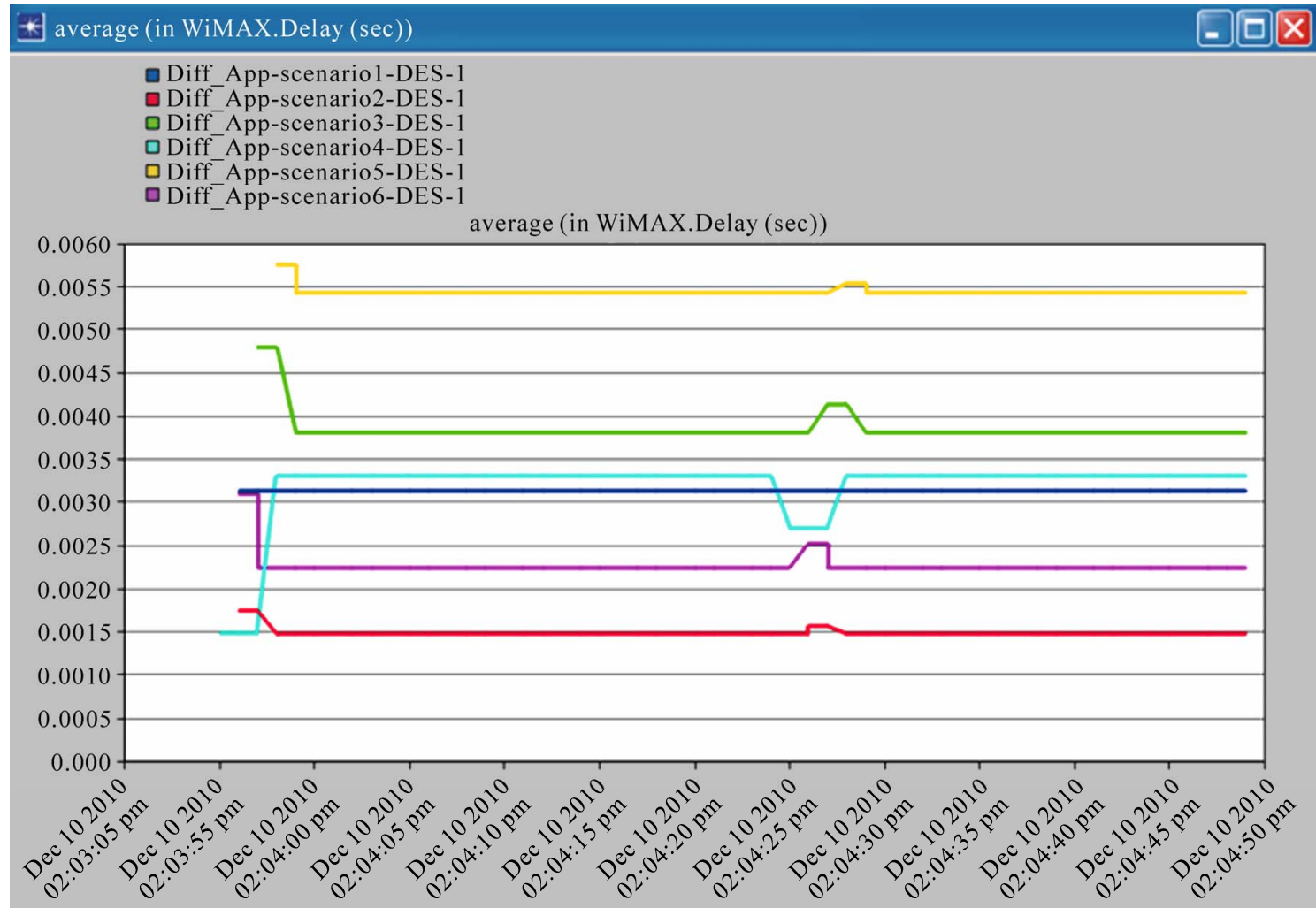

Figure 16. Delay vs. simulation time. 


\section{Pros and Cons}

The whole scenarios are based on the theoretical survey between Jalgaon and Bhusaval. We have observed that the two base stations are well sufficient to cover the desired area. If we increase the base station than somehow load will decrease but cost will increases, since we know that the area covered by one base station is $30 \mathrm{kms}$ (practically) so in our scenario two base stations are efficient.

Our research is dedicated to rural area and his development. In this project we have considered no loss (there are no interference) hence this observation will totally fit with any rural area network.

This research will not fit directly with other city networks. We can apply this concept only when you have good location based survey on that particular area. If we apply this concept on metropolitan city then we should consider about all type of losses.

\section{Conclusions}

This paper presents our location base performance analysis for different application with QoS. Since we know that if the number of nodes in the network increases the throughput of the network becomes high and the delay of the network will reduces in proportionally and it is vice versa for the less number of nodes in general but this will not possible in the case of real or practical environment. In our network models we have observed that there are a lot of factors involved i.e. distance between nodes (Fixed and Mobile) from BS altitude and LOS is different in different scenario with client with location with respect to BS. Since BS is responsible for both DL and UL as well as power consideration so in practical condition result should be affected. In our network the nodes are distributed randomly in every scenario, that's why throughput is not directly reflected (inversely proportional) to delay performance, the same concept is also applied for load. It also shows that WiMAX is really good replacement for existing networks. In this research we had taken fixed node and mobile node as well because this research is totally dedicated to real or practical environment with location based. Our research should help for deployment of WiMAX system to any country including our country India with good QoS to end users or clients. We also observed that QoS has effected with different Application (here Voice over IP Telephony and MPEG).

\section{Future Work}

In the near future, the service providers' motivate to deploy WiMAX in every location because he has very good features for LOS and NLOS. Data Rate (72 Mbps) range $50(\mathrm{Km})$ is few features of WiMAX. The WiMAX service provider wants to increase monthly revenue and improve profitability because the voice revenue is declining. As a result, service providers want to offer advanced services. These services required heavy bandwidth consumers. On the basis of this research they can deploy the networks in all over worlds with efficiently. This research provides the platform for location based security because security is primary concern for any wireless networks.

\section{REFERENCES}

[1] R. K. Jha, A. V. Wankhede and U. D. Dalal, "A Survey of Mobile WiMAX IEEE 802.16 m,” International Journal of Computer Science and Information Security, Vol. 8, No. 1, 2010, pp. 125-131.

[2] IEEE Standard 802.16-2004, “IEEE Standard for Local and Metropolitan Area Networks, Part 16: Air interface for Fixed Broadband Access Systems,” October, 2004.

[3] IEEE Standard 802.16e-2005, "IEEE Standard for Local and Metropolitan Area Networks-Part 16: Air Interface for Fixed and Mobile Broadband Wireless Access Systems Amendment 2,” 28 February 2006.

[4] S. Ramachandran, C. W. Bostian and S. F. Midkiff, "Performance Evaluation of IEEE 802.16 for Broadband Wireless Access,” OPNETWORK, 2002.

[5] V. Rangel, J. Gomez and J. Ortiz, "Performance Analysis of QoS Scheduling in Broadband IEEE 802.16 Based Networks,” OPNETWORK, 2006.

[6] B. L. Dang, Y. Zhao, V. Prasad and I. Niemegeers, "Performance Study of Scheduling Algorithms for WiMAX Networks," OPNETWORK, 2007.

[7] S. Sengupta, M. Chatterjee and S. Ganguly, "Improving Quality of VoIP Streams over WiMAX,” IEEE Transactions on Computers, Vol. 57, No. 2, 2008, pp. 145-156. doi:10.1109/TC.2007.70804

[8] R. K. Jha, A. V. Wankhede and U. D. Dalal, "WiMAX System Simulation and Performance Analysis under the Influence of Jamming," International Journal of Scientific Research, Vol. 1, No. 1, 2010, pp. 20-26.

[9] “OPNET Technologies,” http://www.opnet.com. 